\title{
Improving the Reliability and Availability of Vehicular Communications using Voronoi Diagram-based Placement of Road Side Units
}

\author{
Prithviraj Patil and Aniruddha Gokhale \\ *Vanderbilt University, Dept of EECS \\ Nashville, TN 37212, USA \\ Email: \{prithviraj.p.patil,a.gokhale\}@ vanderbilt.edu
}

\begin{abstract}
Vehicular Ad-hoc Networks (VANETs) form the basis for critical services that improve traffic safety and alleviate traffic congestion. The reliability of VANET-based services and applications that are based solely on vehicle-to-vehicle (V2V) communications, however, is hindered due primarily to limited and often fluctuating V2V communications. To address this limitation, Road-Side Units (RSU) have been proposed to complement V2V communications by providing stable event and data brokering capability. Effective placement of the RSUs is a key requirement in improving reliability of VANET services. This poster describes a novel Voronoi network-based algorithm for the effective placement of RSUs. The reliability metric considered in placing the RSUs involves bounding both the delay incurred by communication packets and packet loss, which in turn ensure timeliness and correct operation of the VANET services.

Index Terms-Vehicular networks; connectivity; placement.
\end{abstract}

\section{INTRODUCTION}

Vehicular Ad-hoc Networks (VANETs) are gaining significant attention due to their potential to support a variety of services of societal and environmental impact [3], [4], e.g. safety of passengers, reducing traffic congestion, driver assistance, etc. Despite the promise offered by VANETs, for these services to indeed be useful to the society and to scale to larger regions and vehicular traffic, VANETs must be able to overcome key challenges stemming from network connectivity bottlenecks in vehicle-to-vehicle $(\mathrm{V} 2 \mathrm{~V})$ communications caused in large part to the highly dynamic nature of the network, and bandwidth loss caused due to high and variable speeds of vehicles moving in different directions [2].

To overcome these challenges, it has been suggested to deploy road-side units (RSUs) as a means to provide a complementary communication capability in the form of a vehicle-toinfrastructure (V2I) network. Of particular importance to us in this poster is improving reliability and availability of network connectivity for VANET services. RSUs help to overcome the inherent unreliability in $\mathrm{V} 2 \mathrm{~V}$ communications by providing an alternate, high availability channel for communication and serving as brokers for data exchange among vehicles, and the vehicles and the wired network.

However, an ad hoc placement of RSUs will not address the original problem. Moreover, a solution comprising RSUs requires an upfront investment, and hence their placement must be planned in accordance to several factors including (a) present and projected traffic patterns and vehicular density, (b) the variety of services that are emerging and their communication profile, and (c) the technical advances as well as limitations of the underlying communication mechanisms. All the while, the cost of placement and maintenance must be kept low for it to be an economically viable solution.

In this poster we address these requirements by proposing a novel RSU placement algorithm based on the notion of a Voronoi Diagram [1]. In our approach the formation of the convex polygons of a Voronoi diagram is determined using traffic density, distance, and probability of V2V connection availability on each road of the chosen region as parameters so that both packet delay and loss are bounded thereby improving the timeliness and reliability of VANET services.

\section{Voronoi Network-BASEd RSU Placement}

This section presents our RSU placement algorithm that is based on the idea of a Voronoi Diagram [1].

\section{A. Basic Idea Behind Our Approach}

Voronoi network (or Voronoi diagram) is defined in [5] as follows: "Let $\mathrm{S}$ be a set of points in Euclidean space with all limit points contained in S. For every point $\mathrm{x}$ in the Euclidean space, there is one point of $S$ closest to x." Let us assume that the RSUs are placed at the location of the generating point of the convex polygons of the Voronoi diagram that are formed for a given urban road network. In such a setup, the contours of the convex polygon that surrounds the RSU will be formed based on whatever criteria we choose to form the polygon. Due to the properties of Voronoi diagram, each vehicle will be now be covered by one RSU only, which also happens to be its nearest RSU from the perspective of packet delay incurred. This criterion maximizes the area covered by given RSUs while meeting the allowable delay bound for communication packets for every vehicle since each vehicle based on its location is assigned to nearest RSU and no vehicle is left unassigned. If this criteria were aggregated with maximizing the number of vehicles served by the RSU, i.e., minimizing the packet losses, then by reconciling these two parameters in the formation of Voronoi diagram, we will have a solution for significantly improving reliability of VANET services. 


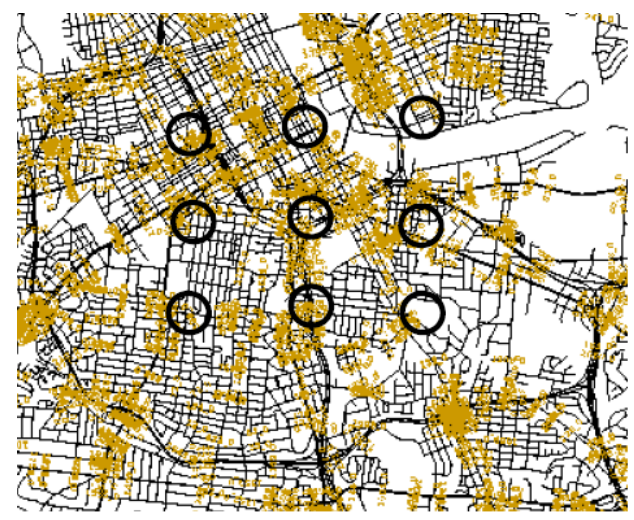

(a) Input to First Phase

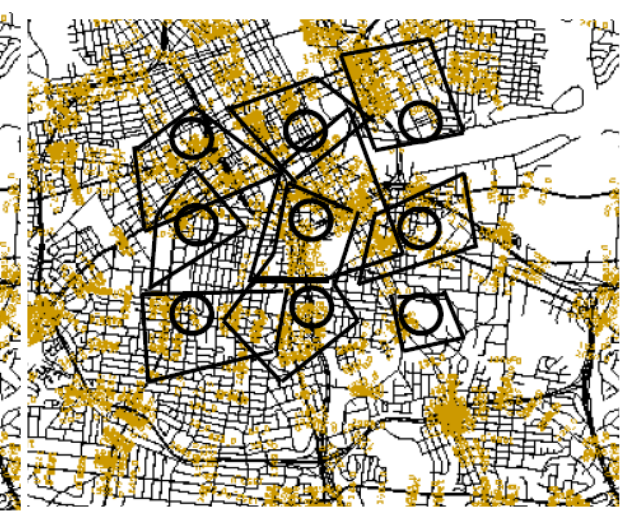

(b) Output of First Phase

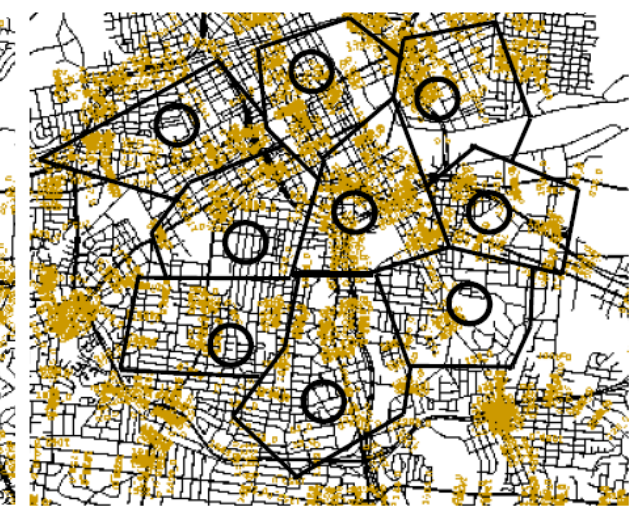

(c) Output of Second Phase

Fig. 1. Maximizing RSU Ranges Shown for 9 RSUs (Circle represents physical range; Polygon represents extended range)

\section{B. Two-Stage Voronoi Diagram Algorithm for VANETs}

Our algorithm works in two stages described below.

1) Stage I Algorithm: Stage I algorithm takes as input the maximum number of RSUs that are allowed by the city planners, a map of an urban area, mobility properties of traffic, and maximum tolerable delay for the service before its quality degrades, and a few other parameters. The algorithm starts by placing RSUs at some initial position either randomly or by spreading them evenly. To determine the density of vehicles in a region, our algorithm uses population density data available from census data and computes these parameters for every RSU. We calculate extended range of RSU by assigning a single packet belonging to the chosen service to all the RSUs in the region and let the packet propagate from a given RSU towards other RSUs by leveraging the vehicles within its signal range (through V2I channel) as well as by hopping from one vehicle to another (through V2V channel).

farthest set of locations that a packet can travel given the mix of vehicles and their speeds along the routes they are allowed to take are computed once the packet delay bound is reached. A set of all such locations represents the extended range of a RSU. The extended range of RSU determines the contours of the polygon while the position of the RSU becomes the generating point. If the extended range of any two pairwise RSUs overlap, then the RSUs in the pair are deemed to be neighbors. Consequently, a neighborhood map of all RSUs for the entire region is computed. Also, each vehicle causes bandwidth loss because of its speed. To take this loss into consideration we compute the portion of total vehicles which respect the delay bound despite bandwidth loss while relaying packet.

2) Stage II Algorithm: Stage II algorithm is an optimization phase which attempts to reduce overlap of a vehicle's connectivity at once to multiple RSU while also minimizing areas of no connectivity. This step is accomplished by moving the position of RSU within its polygon in such a way that a global optimization is realized. Fig 1 shows the input and output for each algorithm stage for a map of urban area with 9 initial RSUs.

\section{Conclusion And Future Work}

We presented an algorithm for RSU placement in vehicular ad hoc networks to improve the reliability of the services offered in such networks. Our algorithm based on Voronoi diagrams adjusts the placement of RSU in accordance to the traffic data populated from census data of Tiger maps of US census bureau. In doing so, it benefits from the traffic patterns and maximizes the normal range of RSU to an extended range. This in turn optimizes the number of RSUs required to achieve a more realiable connectivity and hence improves performance of VANET-based applications. Our initial evaluations show promise with the approach. ${ }^{1}$, however, we carried the experiments with only one service supported in a VANET. For future work, we will focus on extensive evaluations of our approach for different urban settings, traffic patterns and variety of services offered simultaneously. We will also evaluate our algorithm for different quality of service requirements for each individual packet depending on the application.

\section{ACKNOWLEDGMENTS}

This work was supported in part by the National Science Foundation NSF SHF/CNS Award CNS 0915976, and NSF RAPID Awards 1047792 and 1047753. Any opinions, findings, and conclusions or recommendations expressed in this material are those of the author(s) and do not necessarily reflect the views of the National Science Foundation.

\section{REFERENCES}

[1] Franz Aurenhammer. Voronoi diagrams a survey of a fundamental geometric data structure. ACM Comput. Surv., 23:345-405, September 1991.

[2] Dirk Helbing. Traffic and related self-driven many-particle systems. Rev Mod. Phys., 73:1067-1141, Dec 2001.

[3] T. Mangel and H. Hartenstein. An analysis of data traffic in cellular networks caused by inter-vehicle communication at intersections. In Intelligent Vehicles Symposium (IV), 2011 IEEE, pages 473 -478, june 2011.

[4] Sok-Lan Sou. A power-saving model for roadside unit deployment in vehicular networks. Communications Letters, IEEE, 14(7):623 -625, july 2010.

[5] Wikipedia. Voronoi diagram, November 2011.

\footnotetext{
${ }^{1}$ Results are not shown due to space limitations.
} 